\title{
Management of Adult Unilateral TMJ Ankylosis with Temporalis Muscle and Fascia Flap: Review of 51 Cases
}

\author{
Sobhan Mishra, Ramanupam Tripathy, Samrat Sabhlok, Ritesh Roy
}

\begin{abstract}
Purpose: This study is aimed to determine the efficacy of temporalis muscle and fascia flap in the treatment of unilateral temporomandibular joint (TMJ) ankylosis in adults. Treatment of TMJ ankylosis has been a topic of debate and the treatment options are multidimensional. Achieving good postoperative mouth opening and functional and cosmetic outcomes of surgery, depends on the method of reconstruction. The need of study is to give the best possible solutions with minimal morbidity to the underlying deformity.
\end{abstract}

Materials and methods: A retrospective study was done on 51 patients to evaluate the postoperative results for unilateral TMJ ankylosis. Parameters such as etiology, postoperative mouth opening on 1st, 2nd, 6th week and 6 months and intraoperative and postoperative complications were recorded. The operative protocol for unilateral TMJ ankylosis entailed: (1) Resection of the ankylotic mass, (2) intraoral ipsilateral coronoidectomy, (3) contralateral coronoidectomy when necessary, (4) interpositional tissue transfer to the TMJ with temporalis muscle and fascia flap, (5) maxillomandibular fixation (MMF) and (6) early mobilization and aggressive physiotherapy.

Results: The results were encouraging and functional results of interpositional arthroplasty were satisfying with minimal complications. Early postoperative initial aggressive exercise, physiotherapy, and strict follow-up play an important role in preventing postoperative adhesions.

Conclusion: The findings of this study support the use of temporalis muscle and fascia flap in adult patients with unilateral TMJ ankylosis.

Keywords: Temporomandibular joint ankylosis, Temporalis muscle and fascia flap, Interpositional arthroplasty.

How to cite this article: Mishra S, Tripathy R, Sabhlok S, Roy R. Management of Adult Unilateral TMJ Ankylosis with Temporalis Muscle and Fascia Flap: Review of 51 Cases. Int J Head and Neck Surg 2012;3(3):133-136.

Source of support: Nil

Conflict of interest: None

\section{INTRODUCTION}

Ankylosis is a Greek terminology meaning 'stiff joint'. Hypomobility of the joint can lead to inability to open the mouth from partial to complete. Temporomandibular joint (TMJ) ankylosis is a very distressing structural condition that denies the victim the benefit of normal diet and causes substantial facial disfigurement. ${ }^{1-3}$

TMJ ankylosis is most commonly associated with trauma (13-100\%), local or systemic infection (10-49\%) or systemic diseases (10\%), such as ankylosing spondylitis, rheumatoid arthritis and psoriasis. Ankylosis can also occur as a result of TMJ surgery. ${ }^{4}$
The treatment of TMJ ankylosis poses a significant challenge because of technical difficulties of airway and also because of mandibular deficiency. A variety of surgical techniques for its treatment have been described in the literature. However, no single method has produced uniformly successful results. ${ }^{5-7}$

A variety of interposition materials have been used like temporalis muscle and fascia, dermis, auricular cartilage, fascia lata, fat, lyodura, silastic, metals, etc. ${ }^{8-12}$ The most commonly used interposition material is temporalis muscle and fascia flap.

The frequent complications after treatment of ankylosis are restricted mouth oral opening and reankylosis which can be prevented by adequate resection of the ankylotic stump and aggressive postoperative physiotherapy.

In this study, we report a retrospective analysis of the use and effectiveness of temporalis muscle and fascia flap in management of adult unilateral TMJ ankylosis cases.

\section{MATERIALS AND METHODS}

A retrospective evaluation of 51 adult patients, treated between 2007 and 2010 at our center, was done. All patients were medically fit and only adults above 18 years of age were selected for the study. Ethical approval for the study was taken by our institutional review board.

Patients with a chief complaint of restricted mouth opening and interincisal opening of 0 to $15 \mathrm{~mm}$ were selected. All patients were diagnosed as having unilateral TMJ ankylosis on the basis of history, clinical examination orthopantomograph (OPG) and computed tomographic (CT) scan. The age range of the patients was 18 to 35 years.

The preoperative assessment included a thorough medical history and physical examination to determine the ankylosis characteristics according to age group, gender (Table 1), possible causes of ankylosis (Table 2), ankylosis type, treatment, recurrence rate, facial nerve damage after surgery (Table 3), maximal interincisal opening (MIO).

The ankylosis was also classified on operative findings based on Sawhney's classification into two different types, i.e. (1) massive bony outgrowth (Sawhney classification type III, IV) and (2) soft tissue fibrosis (Sawhney classification type I, II; Table 4).

The operative protocol for unilateral TMJ ankylosis in adults was based on Kaban protocol ${ }^{4}$ and consisted of: (1) Resection of ankylotic mass through extended 
Table 1: Gender distribution of patients

\begin{tabular}{lcc}
\hline Gender & No. of patients & Percentage (\%) \\
\hline Male & 39 & 76.4 \\
Female & 12 & 23.6 \\
\hline
\end{tabular}

Table 2: Causes of TMJ ankylosis

\begin{tabular}{lcc}
\hline Cause & No. of patients & Percentage (\%) \\
\hline Falls (simple) & 15 & 29.4 \\
Falls from heights/trees & 30 & 58.8 \\
Dislocated TMJ & 1 & 2 \\
Unknown & 5 & 9.8 \\
\hline
\end{tabular}

Table 3: Operative data of patients

\begin{tabular}{|c|c|c|}
\hline Operative data & No. of patients & Percentage (\%) \\
\hline \multicolumn{3}{|l|}{ Operative findings } \\
\hline Massive bony outgrowth & 42 & 82.3 \\
\hline Soft tissue fibrosis & 9 & 17.7 \\
\hline $\begin{array}{l}\text { Surgery done } \\
\text { Condylectomy, ipsilateral } \\
\text { coronoidectomy, contralateral } \\
\text { coronoidectomy and } \\
\text { temporalis muscle and } \\
\text { fascia flap }\end{array}$ & a & 88.3 \\
\hline $\begin{array}{l}\text { Condylectomy, ipsilateral } \\
\text { coronoidectomy and } \\
\text { temporalis muscle and } \\
\text { fascia flap }\end{array}$ & 6 & 11.7 \\
\hline
\end{tabular}

Table 4: Pre- and postoperative interincisal measurements of patients

\begin{tabular}{lcc}
\hline Interincisal opening & No. of cases & Percentage (\%) \\
\hline Preoperative $(\mathrm{mm})$ & 42 & 82.3 \\
$0-5$ & 6 & 11.7 \\
$6-10$ & 3 & 6 \\
11-15 & & \\
Intraoperative $(\mathrm{mm})$ & 41 & 80.4 \\
$31-40$ & 10 & 19.6 \\
$41-50$ & & \\
One month postoperative $(\mathrm{mm})$ & 12 & 23.5 \\
25-30 & 35 & 68.6 \\
$31-40$ & 4 & 7.8 \\
$41-50$ & & \\
Six months postoperative $(\mathrm{mm})$ & 15 & 29.4 \\
25-30 & 35 & 68.6 \\
$31-40$ & 1 & 2 \\
$41-50$ & & \\
\hline
\end{tabular}

preauricular incision, (2) intraoral ipsilateral coronoidectomy, (3) contralateral coronoidectomy when necessary, (4) the inferiorly based, U-shaped, finger-like temporalis muscle and fascia flap was turned outward and downward over the zygomatic arch and placed in the TMJ, (5) maxillomandibular fixation (MMF) and (6) early mobilization and aggressive physiotherapy. General anesthesia with nasotracheal intubation and total muscle relaxation was administered. A single intravenous dose of steroid was given at the start of the case, generally using 8 to $12 \mathrm{mg}$ of prednisolone. A preauricular incision with a temporal extension was made. The external canal was lightly packed with Vaseline gauze. The temporalis muscle was lifted from the infratemporal fossa toward the anterior at the pericranial level, while the zygomatic root was uncovered. An avascular tissue plane along the cartilaginous meatus was established using surgical scissors. The ankylosed TMJ was palpable and an incision was made directly onto the bone, exposing the ankylosed TMJ. Excision of the fibrous tissue and ankylotic bony mass was carried out using drill and saw. Mouth opening was checked and intraoral contralateral coronoidectomy was carried out wherever required. The TMJ was lined with a temporalis muscle and fascia flap rotated over the arch into the joint. The flap was sutured medially, anteriorly and posteriorly with 4-0 Vicryl. Arch bars were placed following the lining of the joint. Prednisolone was also given intra- and postoperatively (8-12 mg).

Pre- and postoperative interincisal measurements of patients were taken during surgery, after 1 month and again after 6 months.

\section{RESULTS}

This study evaluated 51 patients with an average follow-up of 6 months. Interposition arthroplasty was done on 51 patients. Majority of patients were found to be males $\mathrm{n}=39$ (76.4\%) while the females accounting for $\mathrm{n}=12$ (23.6\%; Table 1). The age group selected for the study was between 18 and 35 years.

Trauma was the most common cause of ankylosis with a positive history of fall from height accounting for 30 patients (58.8\%). Fifteen patients (29.4\%) reported simple falls whereas five patients (9.8\%) could not give any positive history (Table 2).

Intraoperative findings of the joints revealed massive bony union (Sawhney classification III and IV) in 42 patients (82.3\%) and soft tissue fibrous ankylosis (Sawhney classification I and II) in nine patients (17.7\%).

As per the operative protocol by Kaban, all patients with massive bony ankylosis underwent condylectomy. In 45 cases (88.3\%) condylectomy, ipsilateral coronoidectomy and contralateral coronoidectomy was done, whereas in six patients $(11.7 \%)$ contralateral coronoidectomy was not required. In all the cases a temporalis muscle and fascia flap was used as an interposition material.

Of clinical significance in adult TMJ ankylosis patients is the preoperative MIO. Out of 51 patients, 42 patients (82.3\%) had MIO of 0 to $5 \mathrm{~mm}$. In six patients (11.7\%) MIO was 6 to $10 \mathrm{~mm}$ whereas three patients (6\%) had MIO of 11 to $15 \mathrm{~mm}$. 
Forty-one patients (80.4\%) had an intraoperative mouth opening of 31 to $40 \mathrm{~mm}$ while in the other 10 patients (19.6\%) the MIO achieved was up to $50 \mathrm{~mm}$.

All patients were reviewed after 1 month and 35 patients (68.6\%) had an average mouth opening between 31 and $35 \mathrm{~mm}, 12$ patients (23.5\%) had maximal incisal opening between 25 and $30 \mathrm{~mm}$ and four patients (7.8\%) could maintain mouth opening greater than $36 \mathrm{~mm}$.

At 6 months, 35 patients (68.6\%) maintained an average mouth opening of 31 to $35 \mathrm{~mm}$. Fifteen patients (29.4\%) had a visible decreased mouth opening of 25 to $30 \mathrm{~mm}$. only one patient could maintain an MIO of $38.5 \mathrm{~mm}$ (Table 4).

\section{DISCUSSION}

Ankylosis of the TMJ can be classified into true ankylosis (intracapsular) and pseudoankylosis (extracapsular). True ankylosis of the TMJ is defined as any condition that produces fibrous or bony adhesions between the articular surfaces of the TMJ.

True ankylosis is most commonly associated with trauma or infection. ${ }^{13,14}$ Chandra and Dave ${ }^{13}$ reported 258 cases and found $67.8 \%$ associated with trauma and $17 \%$ with infection. Haider ${ }^{14}$ reported eight cases, all of which were associated with trauma. In the present study, trauma (85.3\%) was the major cause of unilateral TMJ ankylosis in the adult patients.

Diagnosis was performed by physical examination and proper radiological examination. ${ }^{15}$ The patient may have difficulty in opening the mouth as well as malocclusion. The degree of limitation in mandibular motion varies considerably and depends on the type and amount of tissue connecting the mandible. ${ }^{16}$

Management of TMJ ankylosis entails surgical intervention. The need to use an interpositional material to prevent TMJ reankylosis after arthroplasty in treatment of TMJ ankylosis has been widely discussed. A variety of interposition materials have been used, including temporalis muscle and fascia, dermis, auricular cartilage, fascia lata, fat, lyodura, silastic, silicone and various metals $\mathrm{s}^{3,8,9,11,12,17-19}$ The most commonly used interposition material at present is temporalis muscle flap. ${ }^{9,20}$

The temporalis muscle and fascia flap have been widely used for the reconstruction of the TMJ. Their principal advantages are their autogenous nature, resilience, adequate blood supply and proximity to the joint, allowing for a pedicled transfer of vascularized tissue into the joint area. ${ }^{21-24}$ Temporalis fascia, with a temporalis muscle varying in thickness, may be harvested as an axial flap based on the middle and deep temporal arteries and veins. ${ }^{23}$

Pogrel and $\mathrm{Kaban}^{23}$ described a flap that may include only fascia or both fascia and muscle, and is rotated inferiorly over the zygomatic arch and into the joint space. They suggested reducing the thickness of the zygomatic arch, if necessary, to avoid bulkiness.

Bergey and Braun ${ }^{25}$ described how the posterior zygomatic arch can be osteotomized in two sites and a segment of the arch removed. The flap then may be rotated through the space created by the osteotomy into the joint and the segment of the arch replaced and secured with rigid fixation.

Kaban et $\mathrm{al}^{4}$ recommended a management protocol for TMJ ankylosis consisting of aggressive resection, ipsilateral coronoidectomy, contralateral coronoidectomy when necessary, lining of the TMJ with temporalis fascia or cartilage, reconstruction of the ramus with a costochondral graft, rigid fixation, and early mobilization and aggressive physiotherapy. The protocol was retrospectively evaluated in the first 14 patients (18 TMJs) treated and followed up postoperatively for at least 1 year. The facial asymmetries present in all unilateral cases remained corrected. The mean maximum postoperative interincisal opening at 1 year was $37.5 \mathrm{~mm}$, lateral excursions were present in 16 of 18 joints (vs none of the 18 joints preoperatively), and pain was present in two of 18 joints (vs 13 of 18 preoperatively). The results of this study indicate that this protocol is effective for treatment of TMJ ankylosis.

In the present study, 51 patients with follow-up periods longer than 6 months manifested MIO distance of $30 \mathrm{~mm}$ or more in 36 cases.

Recurrence is a major problem that occurs after the release of TMJ ankylosis. ${ }^{9}$ Raveh et $\mathrm{al}^{26}$ proposed that the radical removal of the TMJ bone is essential to avoid recurrence. Chossegros et $\mathrm{al}^{9}$ on the other hand, reported that early physiotherapy and choice of interpositional material are important in preventing recurrence.

The immediate postoperative period is the most critical time for successful treatment of TMJ ankylosis. Postoperative pain medications, vigorous physiotherapy and CPM therapy are used to maintain the mobility obtained during surgery and to prevent postsurgical hypomobility secondary to fibrous adhesions. ${ }^{15}$

The tongue blades were used for exercise while the ratchet mouth props were used to count the number of blades during each appointment. ${ }^{15}$ All patients underwent physiotherapy consisting of active and passive ranges of motion exercise at least once a day for the first 2 weeks and once every 2 days for the following 2 weeks. The patient's diet at this time consisted of very soft foods. During the next 3 to 4 weeks the diet was gradually advanced to a solid consistency. As the diet regimen is advanced from a soft to a regular diet, patients are informed about the guidelines to assist them in making sure they do not overuse their jaw. 


\section{CONCLUSION}

The goal of temporomandibular ankylosis is two-fold. One is to achieve adequate mouth opening and second is to prevent recurrence of ankylosis. Functional and esthetic outcome of the surgery depends on the previous anatomy, surgical plan and follow-up physiotherapy. Interpositional arthroplasty using locally available temporalis muscle and fascia proves to be a valuable tool to fill the surgical defect and also to prevent recurrence. It also obviates the disadvantages of alloplastic materials as well as nonvascularized autogenous tissues.

Early postoperative physiotherapy, strict follow-up are essential to prevent postoperative adhesions. This study proves and stresses upon the need for effective solution for management of adult TMJ ankylosis. It can safely be concluded that temporalis muscle and fascia proves to be an effective solution for TMJ ankylosis.

\section{REFERENCES}

1. El-Sheikh MM, Medra AM. Management of unilateral temporomandibular ankylosis associated with facial asymmetry. J Craniomaxillofac Surg 1997;25:109-15.

2. Guthua SW, Maina DM, Kahugu M. Management of posttraumatic temporomandibular joint ankylosis in children: case report. East Afr Med J 1995;72:471-75.

3. Karaca C, Barutcu A, Menderes A. Inverted, T-shaped silicone implant for the treatment of temporomandibular joint ankylosis. J Craniofac Surg 1998;9:539-42.

4. Kaban LB, Perrott DH, Fisher K. A protocol for management of temporomandibular joint ankylosis. J Oral Maxillofac Surg 1990;48:1145-51.

5. Roychoudhury A, Parkash H, Trikha A. Functional restoration by gap arthroplasty in temporomandibular joint ankylosis: A report of 50 cases. Oral Surg Oral Med Oral Pathol Oral Radiol Endod 1999;87:166-69.

6. Su-Gwan K. Treatment of temporomandibular joint ankylosis with temporalis muscle and fascia flap. Int J Oral Maxillofac Surg 2001;30:189-93.

7. Gliven O. Treatment of temporomandibular joint ankylosis by a modified fossa prosthesis. J Craniomaxillofac Surg 2004;32(4):236-42.

8. Chossegros C, Guyot L, Cheynet F, Blanc JL, et al. Comparison of different materials for interposition arthroplasty in treatment of temporomandibular joint ankylosis surgery: Long term follow-up in 25 cases. Br J Oral Maxillofac Surg 1997;35: 157-60.

9. Chossegros C, Guyot L, Cheynet F, et al. Full-thickness skin graft interposition after temporomandibular joint ankylosis surgery. A study of 31 cases. Int J Oral Maxillofac Surg 1999;28: 330-34.

10. Feinberg SE, Larsen PE. The use of a pedicled temporalis muscle-pericranial flap for replacement of the TMJ disc: Preliminary report. J Oral Maxillofac Surg 1989;47:142-46.

11. Matukas VJ, Lachner J. The use of autologous auricular cartilage for temporomandibular joint disc replacement: A preliminary report. J Oral Maxillofac Surg 1990;48:348-53.

12. Meyer RA. The autogenous dermal graft in temporomandibular joint disc surgery. J Oral Maxillofac Surg 1988;46:948-54.
13. Chandra P, Dave PK. Temporomandibular joint ankylosis. Prog Clin Biol Res 1985;187:449-58.

14. Haider Z. Ankylosis of the temporomandibular joint: Causes and management. J Oral Med 1986;41:246-49.

15. Spijkervet FKL, De Bont LGM, Boering G. Management of pseudoankylosis of the temporomandibular joint: Report of cases. J Oral Maxillofac Surg 1994;52:1211-17.

16. Fonseca RJ, Walker RV. Oral and maxillofacial trauma. Philadelphia: WB Saunders 1991;504.

17. Paterson AW, Shepherd JP. Fascia lata interpositional arthroplasty in the treatment of temporomandibular joint ankylosis caused by psoriatic arthritis. Int J Oral Maxillofac Surg 1992;21:137-39.

18. Schoeller T, Hussl H. A modified technique for temporalis transfer. Br J Plastic Surg 1996;49:573-74.

19. Wolfe SA. Rediscovery of the temporalis muscle flap for ankylosis. J Oral Maxillofac Surg 1990;48:771-72.

20. Habel G, Hensher R. The versatility of the temporalis muscle flap in reconstructive surgery. Br J Oral Maxillofac Surg 1986;24:96-101.

21. Clauser L, Curioni C, Spanio S. The use of temporalis muscle flap in facial and craniofacial reconstructive surgery. A review of 182 cases. J Craniomaxillofac Surg 1995;23:203-14.

22. Omura S, Fujita K. Modification of the temporalis muscle and fascia flap for the management of ankylosis of the temporomandibular joint. J Oral Maxillofac Surg 1996;54: 794-95.

23. Pogrel MA, Kaban LB. The role of a temporalis fascia and muscle flap in temporomandibular joint surgery. J Oral Maxillofac Surg 1990;48:14-19.

24. Smith JA, Sandler NA, Ozaki WH, et al. Subjective and objective assessment of the temporalis myofascial flap in previously operated temporomandibular joints. J Oral Maxillofac Surg 1999;57:1058-65.

25. Bergey DA, Braun TW. The posterior zygomatic arch osteotomy to facilitate temporalis flap placement. J Oral Maxillofac Surg 1994;52:426-27.

26. Raveh J, Vuillemin T, Ladrach K, et al. Temporomandibular joint ankylosis: Surgical treatment and long-term results. J Oral Maxillofac Surg 1989;47:900-06.

\section{ABOUT THE AUTHORS}

\section{Sobhan Mishra}

Professor and Head, Department of Oral and Maxillofacial Surgery Institute of Dental Sciences, Director, Kimaaya Cleft and Craniofacial Centre, SSB Hospital, Bhubaneshwar, Odisha, India

\section{Ramanupam Tripathy}

Reader, Department of Oral and Maxillofacial Surgery, Institute of Dental Sciences, Bhubaneshwar, Odisha, India

\section{Samrat Sabhlok (Corresponding Author)}

Senior Lecturer, Department of Oral and Maxillofacial Surgery Dr DY Patil Dental College and Hospital, Pune, Maharashtra, India Phone: 91-8308059830,e-mail: samratsabhlok@yahoo.com

\section{Ritesh Roy}

Consultant, Department of Anesthesia, Kimaaya Cleft and Craniofacial Centre SSB Hospital, Bhubaneshwar, Odisha, India 